\section{(2) OPEN ACCESS}

\title{
Diagnosis and management of bile acid diarrhoea: a survey of UK expert opinion and practice
}

\author{
Julian R F Walters (D) , 1,2 Ramesh Arasaradnam (D) , \\ $\mathrm{H}$ Jervoise N Andreyev, ${ }^{4,5}$ for the UK Bile Acid Related Diarrhoea \\ Network
}

${ }^{1}$ Department of

Gastroenterology, Imperial

College Healthcare NHS Trust, London, UK

2Division of Digestive Diseases, Imperial College London, London, UK

${ }^{3}$ Department of Gastroenterology, University Hospitals Coventry and Warwickshire NHS Trust,

Coventry, UK

${ }^{4}$ Department of

Gastroenterology, Lincoln County Hospital, Lincoln, UK

${ }^{5}$ School of Medicine, University of Nottingham, Nottingham, United Kingdom

Correspondence to Professor Julian R F Walters, Gastroenterology, Imperial College Healthcare NHS Trust, London, UK; julian.walters@ imperial.ac.uk

Received 27 July 2019 Revised 16 August 2019 Accepted 2 September 2019 Published Online First 11 September 2019

\section{Check for updates}

(c) Author(s) (or their employer(s)) 2020. Re-use permitted under CC BY-NC. No commercial reuse. See rights and permissions. Published by BMJ.

\section{To cite: Walters JRF,}

Arasaradnam $\mathrm{R}_{\text {, }}$

Andreyev HJN, et al.

Frontline Gastroenterology

2020:11:358-363.

\begin{abstract}
Objective Bile acid diarrhoea (BAD), which includes bile acid malabsorption, causes a variety of digestive symptoms. Diagnostic rates and management vary considerably. We conducted a survey of current practice to review expert opinion and provide guidance on diagnosis and management.
\end{abstract}

Design/method An online survey was conducted of clinical members of the UK Bile Acid Related Diarrhoea Network, who had all published research on BAD $(n=21)$. Most were National Health Service consultants who had diagnosed over 50 patients with the condition. Results The preferred terminology was to use BAD, with primary and secondary to classify causes. A wide range of presenting symptoms and associated conditions were recognised. SeHCAT (tauroselcholic acid) was the preferred diagnostic test, and $50 \%$ of respondents thought general practitioners should have access to this. Patients who met the Rome IV diagnostic criteria for functional diarrhoea, irritable bowel syndrome (IBS) with predominant diarrhoea or postcholecystectomy diarrhoea were usually investigated by SeHCAT, which was used sometimes in other types of IBS. Treatment with a bile acid sequestrant was offered to patients with low SeHCAT values, with expected response rates $>70 \%$ in the most severe. Colestyramine was the usual sequestrant, starting between $2 \mathrm{~g}$ and $8 \mathrm{~g}$ daily; colesevelam was an alternative. In patients who had an incomplete response, increasing the dose, changing to an alternative sequestrant, use of loperamide and a low fat diet were suggested. Recommendations for follow-up and to improve the overall patient experience were made.

Conclusion This expert survey indicates current best practice in the diagnosis and management of BAD.

\section{Significance of this study}

What is already known on this topic

- Bile acid diarrhoea is common but is often undiagnosed.

- Treatment of the condition is variable.

What this study adds

- Experts recommend the use of SeHCAT testing in functional diarrhoea, irritable bowel syndrome with predominant diarrhoea (IBS-D) and postcholecystectomy diarrhoea.

- Treatment with bile acid sequestrants is predicted to be successful in most patients with a positive SeHCAT test.

- Strategies to improve treatment and the overall experience are suggested.

How might it impact on clinical practice in the foreseeable future

- This review of expert opinion may help improve the diagnostic rates and management of people with bile acid diarrhoea.

- Improved diagnosis and treatment will particularly impact the large proportion of patients with IBS-D or functional diarrhoea who actually have bile acid diarrhoea.

\section{INTRODUCTION}

Excess bile acids in the colon are increasingly recognised as causing a wide spectrum of gastrointestinal symptoms, in particular intermittent or persistent diarrhoea, but also in many patients, bowel frequency, urgency, nocturnal defaecation, excessive flatulence, abdominal pain and sometimes faecal incontinence. ${ }^{1-4}$ This condition has been termed bile acid diarrhoea (BAD), bile acid malabsorption (BAM) or bile salt malabsorption and has 


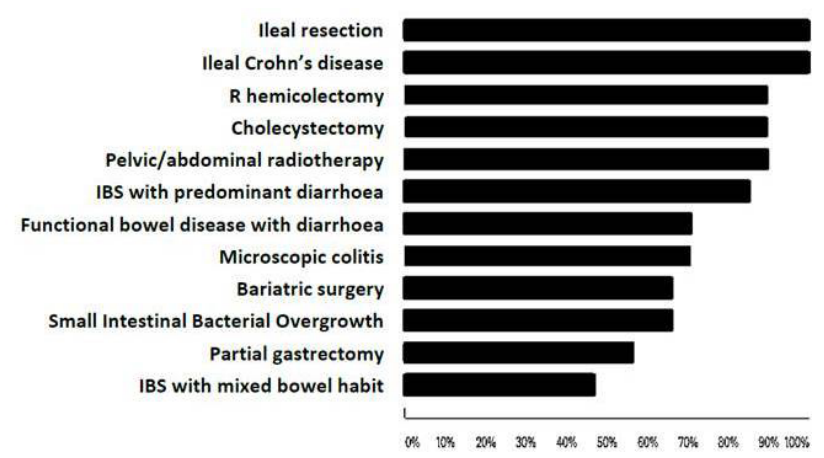

Figure 1 Conditions recognised as associated with a higher incidence of bile acid diarrhoea. The percentage of respondents who identified specific conditions as associations with bile acid diarrhoea is shown. IBS, irritable bowel syndrome.

also been known as cholerheic enteropathy when first described over 50 years ago. ${ }^{5}$

Following ileal resection, or sometimes in other gastrointestinal disorders such as active Crohn's disease, during cancer chemotherapy or after pelvic irradiation, the reabsorption of conjugated bile salts in the terminal ileum is decreased, resulting in excess colonic bile acids producing fluid and electrolyte secretion. A clinical response occurs to bile acid sequestrants (BAS), including colestyramine, colestipol or colesevelam. ${ }^{6-9}$ In patients with no cause for secondary BAM, primary or idiopathic BAD is common and forms a subset of those diagnosed with chronic functional diarrhoea or irritable bowel syndrome with predominant diarrhoea (IBS-D). ${ }^{3}{ }^{10}{ }^{11}$ Most of these patients have no defect in bile acid absorption. The mechanism of this condition is hepatic overproduction of bile acids, as shown by increased blood concentrations of the bile acid precursor $7 \alpha-\mathrm{OH}-4$-cholesten-3-one (C4) and secondary to impaired feedback by the ileal hormone fibroblast growth factor 19 (FGF19). ${ }^{12}$

A survey by patient groups has shown that there is poor recognition by professionals, with diagnostic delay often exceeding 5 years, and a large unmet need in symptom control. ${ }^{13}$ There is no diagnostic method for BAD with worldwide availability, although a comparison of different biomarker studies, including measurements of C4 or FGF19, ${ }^{14}$ has shown similar detection rates in IBS-D and functional diarrhoea from many centres. ${ }^{15}$ Faecal bile acid collections are unpopular but have been optimised by Camilleri and colleagues at the Mayo Clinic to include the percentage of primary bile acids. ${ }^{16}$ The SeHCAT test, which uses ${ }^{75}$ Se-radiolabelled 23-Selena-25-homotaurocholate (tauroselcholate), was developed in the $1980 \mathrm{~s}^{17}$ and has been shown to predict the response to therapies including colestyramine, ${ }^{18}$ colestipol, ${ }^{8}$ colesevelam, ${ }^{19}$ a low fat $\operatorname{diet}^{20}$ and obeticholic acid. ${ }^{21}$ Use of SeHCAT in the UK has grown substantially, particularly in the last decade (GE Healthcare, personal communication), and has been reviewed. ${ }^{22}{ }^{23}$ However, SeHCAT is only available in certain countries and is not licenced in the USA; this has greatly impaired the understanding of its value and so hindered recognition of $\mathrm{BAD}$, development of new drugs and formulation of consensus guidelines.

Diagnostic rates and management vary considerably even in the UK. ${ }^{23}$ The UK Bile Acid Related Diarrhoea Network (UK-BARDN) was established in 2017 as a forum for researchers with recent publications on BAD/BAM. We now report a survey of current practice, reviewing expert opinion and providing further guidance on diagnosis and management.

\section{METHODS}

We identified areas of debate in the diagnosis and management of BAD and BAM. We developed a survey, which comprised 24 questions (for full details see online supplementary data). Answers were collected on Survey Monkey and were analysed.

The link to the survey was sent at the end of 2018 to 21 clinicians in UK-BARDN. At the time of the survey, 95\% held a consultant post in the NHS; this is now $100 \%$. A response rate of $100 \%$ was obtained. Respondents estimated the number of patients they had diagnosed. This was over 50 in $85 \%$ and more than 100 patients in $48 \%$.

Where appropriate, comparisons of proportions per group were made by Fisher's exact test. A p value of 0.05 was taken as significant.

\section{RESULTS}

\section{Terminology}

As the terminology for this condition has varied, we wanted to see if there was a majority for specific usage. 'Bile acid diarrhoea' was the first preference for $57 \%$, 'bile acid malabsorption' for 14\%, with 29\% using either term depending on the clinical circumstances. In classifying the different types, 'primary or secondary' was the first preference for $89 \%$. Second preference was for 'types 1, 2 and $3,{ }^{24}$ with 'overproduction or malabsorption" ${ }^{12}$ the third choice.

\section{Associated conditions}

We asked whether certain specific conditions were recognised as associated with a higher incidence of BAD. Figure 1 shows that the majority of respondents identified ileal resection, Crohn's disease, right hemicolectomy, cholecystectomy, pelvic or abdominal radiotherapy, IBS with predominant diarrhoea, functional bowel disease with diarrhoea, microscopic colitis, bariatric surgery, small intestinal bacterial overgrowth and partial gastrectomy as associations.

\section{Symptoms}

Specific symptoms that would lead to respondents considering a diagnostic test for BAD are shown in table 1 . Loose stools and frequency greater than three times/day were the most common symptoms, but 
Table 1 Symptoms routinely leading to consideration of a diagnostic test for bile acid diarrhoea

\begin{tabular}{ll}
\hline Symptoms & $\%$ of respondents \\
\hline Always having loose stools & 86 \\
Frequency of bowel movements $>6 \times /$ day & 86 \\
Frequency of bowel movements 3-6x/day & 81 \\
Intermittent loose stools & 76 \\
Faecal urgency at least weekly & 67 \\
Faecal incontinence at least weekly & 62 \\
\hline
\end{tabular}

intermittent loose stools, faecal urgency and incontinence were also recognised by most responders. Symptoms that the majority did not routinely consider a test included weight gain or loss combined with loose stools, variable loose and hard stools, anal faecal soiling, frequency between 1 and 3 times/day, abdominal pain associated with or relieved by defaecation or specific yellow or green coloured stools.

\section{Diagnostic tests}

All respondents selected SeHCAT as a diagnostic test for BAD. Blood tests, specifically FGF19 and C4, were recognised by $61 \%$ and $57 \%$ and a therapeutic trial (as a diagnostic test) by $47 \%$. SeHCAT was the test used most frequently, by $95 \%$, with a therapeutic trial the second choice for $85 \%$. Access to SeHCAT, FGF19, C4 and faecal tests in hospitals was thought appropriate. Access by general practitioners (GPs) to SeHCAT testing was proposed by $50 \%$. It was recognised that hospitals and GPs would also conduct therapeutic trials.

The next questions were about the actual use of these tests in specific clinical situations and were limited to SeHCAT and a therapeutic trial, as other tests were used infrequently (table 2).

Reflecting the wording in the current Rome IV criteria, in functional diarrhoea, SeHCAT would be requested usually (ie, $>70 \%$ of the time) by $80 \%$ of the respondents. This was significantly more than the $10 \%$ who would usually use a therapeutic trial $(\mathrm{p}<0.001)$. Similarly in IBS-D, SeHCAT was usually requested by $70 \%$ of the respondents $(\mathrm{p}<0.002$ compared with trial). In IBS with mixed bowel habit, SeHCAT was usually requested by $43 \%$, at least sometimes (>30\% of the time) by $62 \%$, but rarely by $38 \%$. In IBS with constipation, SeHCAT was rarely used by $50 \%$.

In postcholecystectomy diarrhoea, SeHCAT was usually used by $71 \%$ but $36 \%$ who would usually use a trial $(\mathrm{p}=0.08)$. Including those who would use these

Table 2 Use of SeHCAT or therapeutic trial to diagnose bile acid diarrhoea in specific clinical situations

\begin{tabular}{|c|c|c|c|c|c|c|c|c|c|c|}
\hline \multirow[b]{3}{*}{ Condition } & \multicolumn{10}{|c|}{ Proportion requesting test (\%) } \\
\hline & \multicolumn{5}{|l|}{ SeHCAT } & \multicolumn{5}{|c|}{ Therapeutic trial } \\
\hline & Always & Usually & Sometimes & Rarely & Never & Always & Usually & Sometimes & Rarely & Never \\
\hline \multicolumn{11}{|l|}{$\begin{array}{l}\text { 'How often would you request [this test], in a } \\
\text { patient without other significant findings on } \\
\text { history or examination ...' }\end{array}$} \\
\hline \multicolumn{11}{|l|}{$\begin{array}{l}\text { '... with episodic diarrhoea for }>6 \text { months, } \\
\text { without predominant abdominal pain or } \\
\text { bothersome bloating ...' }\end{array}$} \\
\hline $\begin{array}{l}\text { '... with }>25 \% \text { of the stools in the last } 3 \\
\text { months being BSFS type } 6 / 7 \text { ?' }\end{array}$ & 65 & 15 & 15 & 5 & 0 & 0 & 10 & 20 & 20 & 30 \\
\hline $\begin{array}{l}\text { '... with }>25 \% \text { of the stools being BSFS type } \\
6 / 7 \text { starting after cholecystectomy?' }\end{array}$ & 38 & 33 & 19 & 10 & 0 & 7 & 29 & 14 & 29 & 21 \\
\hline \multicolumn{11}{|l|}{$\begin{array}{l}\text { '... in Crohn's disease with diarrhoea, ileal } \\
\text { resection of } 50-100 \mathrm{~cm} \text {, and ...' }\end{array}$} \\
\hline '...negative inflammatory markers?' & 32 & 16 & 26 & 11 & 16 & 31 & 19 & 25 & 13 & 13 \\
\hline '... raised inflammatory markers?' & 21 & 11 & 21 & 11 & 38 & 7 & 0 & 14 & 36 & 43 \\
\hline \multicolumn{11}{|l|}{ ' $\ldots$ with features of IBS* $\ldots$ ' } \\
\hline $\begin{array}{l}\text { '... with episodic diarrhoea in the last } 3 \text { months, } \\
\text { with }>25 \% \text { of stools type } 6 / 7 \text {, and }<25 \% \text { type } \\
1 / 2 \text { ?' }\end{array}$ & 45 & 25 & 25 & 5 & 0 & 0 & 14 & 7 & 36 & 43 \\
\hline $\begin{array}{l}\text { '... with variable bowel habit in the last } 3 \\
\text { months, with }>25 \% \text { of stools type } 6 / 7 \text {, and } \\
>25 \% \text { type } 1 / 2 \text { ?' }\end{array}$ & 19 & 24 & 19 & 29 & 10 & 0 & 7 & 31 & 0 & 62 \\
\hline $\begin{array}{l}\text { '... with variable bowel habit in the last } 3 \\
\text { months, with }<25 \% \text { of stools type } 6 / 7 \text {, and } \\
>25 \% \text { type } 1 / 2 \text { ?' }\end{array}$ & 20 & 15 & 15 & 25 & 25 & 0 & 0 & 14 & 14 & 71 \\
\hline
\end{tabular}

Inflammatory markers=serum $\mathrm{C}$ reactive protein and faecal calprotectin.

The following figures were suggested as an indication of the estimated frequency:.

'Always' =>99\%; 'Usually' $=>70 \%$; 'Sometimes' $=30 \%-70 \%$; 'Rarely' $=<30 \%$; 'Never' $=<1 \%$.

*Features of IBS=abdominal pain> $>1$ day/week, related to defaecation and associated with change in frequency or form of stool for $>6$ months.

BSFS, Bristol Stool Form Scale; IBS, irritable bowel syndrome. 


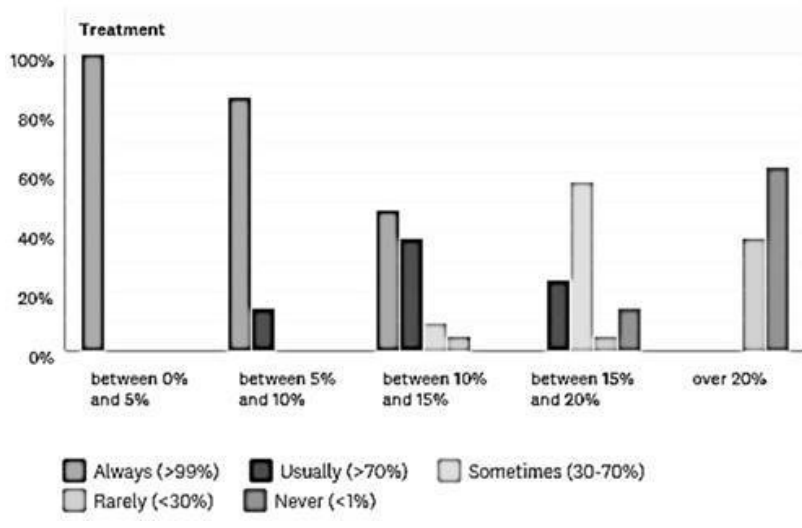

Figure 2 Likelihood of treatment according to SeHCAT result. Percentages of respondents who would offer treatment to patients in SeHCAT categories of 'between $0 \%$ and $5 \%$ ', 'between $5 \%$ and $10 \%$ ', 'between 10\% and 15\%', 'between 15\% and 20\%' and 'over 20\%' are shown. Respondents were asked to classify their likelihood of treatment as 'always' (>99\%), 'usually' (>70\%), 'sometimes' (30\%$70 \%)$, 'rarely' (<30\%) or 'never' $(0 \%)$.

sometimes, SeHCAT use was significantly greater than using a trial $(90 \%$ vs $50 \%, \mathrm{p}=0.02)$.

In patients with Crohn's disease with diarrhoea, ileal resection of $50-100 \mathrm{~cm}$, and negative inflammatory markers, a therapeutic trial would be used roughly as often as SeHCAT by half the respondents. Either was considered at least sometimes by at least $70 \%$. If inflammatory makers were also raised, SeHCAT was used by over $50 \%$ at least sometimes. However, use of a trial was much less likely than when the inflammatory markers were negative $(p=0.02)$.

\section{Management}

The likelihood of offering treatment varied according to the SeHCAT result, as shown in figure 2. All respondents would always treat patients with a 7-day retention between $0 \%$ and $5 \%$, defined as severe disease. With moderate disease (SeHCAT 5\%-10\%), most $(86 \%)$ would also always treat, and the rest would usually do so. Most (86\%) would usually treat mild disease (10\%-15\%) and $80 \%$ would sometimes offer treatment to those with a borderline SeHCAT (15\%-20\%). Treatment of those with normal SeHCAT results (>20\%) was rarely offered.
We asked what response rate would be expected, based on the respondents' experience, following optimisation of BAS therapy, in a patient with specific SeHCAT result (table 3 ). The estimated response rate varied inversely with SeHCAT test values.

Colestyramine was the first-line BAS used by $95 \%$. Starting doses for a $70 \mathrm{~kg}$ women varied, with $29 \%$ using $2 \mathrm{~g}$ once daily, $38 \% 4 \mathrm{~g}$ once daily, $24 \% 4 \mathrm{~g}$ twice daily and $10 \% 4 \mathrm{~g}$ three times daily. Colesevelam was also used, with $29 \%$ starting with $625 \mathrm{mg}$ once daily, $24 \%$ with $1.25 \mathrm{~g}$ once daily, $29 \% 1.25 \mathrm{~g}$ twice daily and $10 \% 1.25 \mathrm{~g}$ three times daily or another regime. There was a preference to give these drugs last thing at night (by 38\%) rather than with food (by 29\%). Warnings to take other medications 1 hour before or 4 hours after the BAS, in order to avoid interactions, were usually given by $76 \%$.

In patients who had an incomplete response, common recommendations included increasing the dose (100\% of respondents), increasing use of drugs such as loperamide (80\%), changing to an alternative BAS $(71 \%)$ and advice on a low fat $(40 \mathrm{~g} / \mathrm{d}) \operatorname{diet}(71 \%)$. Avoidance of high FODMAP foods was recommended by $29 \%$. There was little difference in these recommendations whether the patient had a SeHCAT result of $3 \%$ or $13 \%$.

In the follow-up of a typical patient treated with a BAS, annual review by a specialist or GP, patient support groups, dietetic and pharmacist review and monitoring of blood vitamins and lipids were all given broadly similar importance.

To improve the overall patient experience, greater recognition by various professional groups, in particular gastroenterologists, in the popular press, by GPs, with improved diagnosis and drugs, were all considered important by the majority of respondents.

\section{DISCUSSION}

This survey of experts represents the experience of over 20 British gastroenterologists who have treated in excess of 1000 patients with BAD. It provides a review of current best practice in the diagnosis and management of patients with $\mathrm{BAD}$, identifying where there is consensus and where there are uncertainties that need further investigation.

Table 3 Predicted percentage response rates to bile acid sequestrant therapy according to SeHCAT result

\begin{tabular}{lccccc}
\hline & \multicolumn{3}{l}{ Predicted response rate } & & \\
\cline { 2 - 6 } SeHCAT 7d retention & $>90 \%$ & $70 \%-90 \%$ & $50 \%-70 \%$ & $30 \%-50 \%$ & 0 \\
\hline $0 \%-5 \%$ & 53 & 48 & 0 & 0 & 5 \\
$5 \%-10 \%$ & 24 & 58 & 14 & 29 & 5 \\
$10 \%-15 \%$ & 5 & 14 & 48 & 45 & 45 \\
$15 \%-20 \%$ & 0 & 0 & 10 & 0 & 100 \\
\hline $20 \%$ & 0 & 0 & 0 & & 5 \\
\hline
\end{tabular}

The percentage of respondents choosing each result is shown. 
There was general consensus on the conditions that predispose to BAD and malabsorption. Ileal resection or right hemicolectomy, Crohn's disease, postcholecystectomy diarrhoea, abdominal or pelvic radiotherapy and functional bowel disease with diarrhoea, including IBS-D, were recognised. The need to make a diagnosis with SeHCAT testing was clearly demonstrated. Only in the group of patients with Crohn's disease, ileal resection and negative inflammatory markers did a trial of therapy receive support similar to that given for SeHCAT. Presumably, this was because of the accumulated data that has shown a greater than 90\% likelihood of these patients having an abnormal SeHCAT. ${ }^{25} 26$ Recognition of the association of symptoms of BAD with microscopic colitis, bariatric surgery, small intestinal bacterial overgrowth and partial gastrectomy are also noted.

SeHCAT testing has been more widely used in the UK than most other countries. The accumulated experience here is important in informing diagnostic decision making elsewhere. The need to consider BAD in functional bowel disorders with diarrhoea has gained greater awareness in the latest Rome IV guidance, ${ }^{3}$ and we now provide data to expand on this. In our study, experts consider SeHCAT testing is usually necessary in patients meeting the current criteria for functional diarrhoea and for IBS with predominant diarrhoea. Experts considered this sometimes also in patients with IBS and mixed bowel habits. This reflects recent guidance on investigation of chronic diarrhoea from the British Society of Gastroenterology published recently. ${ }^{4}$ Possibly GPs should have access to this test, as was suggested here, and this would help increase the recognition and reduce the diagnostic delay highlighted in a recent patient survey. ${ }^{13}$ However, a more consistent practice throughout the UK by gastroenterologists will benefit patients and healthcare delivery. ${ }^{22} 23$ Recent studies have shown that early SeHCAT testing to diagnose BAD has significant economic advantages, with a reduction in unnecessary other tests, in particular cross-sectional imaging, repeated colonoscopies and unnecessary trials of expensive medications. ${ }^{27} 28$

It is likely that, internationally, rates of BAD do not vary greatly, ${ }^{215}$ but lack of availability of SeHCAT in the USA and many other countries is a problem. If SeHCAT licencing cannot be extended, then other tests such as 48 hours total or primary faecal bile acids, C4 and FGF19, will require further development and validation, as has been recently shown at the Mayo Clinic. ${ }^{16}$ Experience of these tests by the current respondents was limited and so was not a major consideration in this survey. Previous findings suggest particularly that the negative predictive values of low C4 and high FGF19 can both have a role in the diagnostic pathway of BAD and may help in the selection of people for further testing. ${ }^{14}$ Estimation of the proportion of faecal primary bile acids is an interesting development related to bile acid secretion, absorption and metabolism, but its value needs further development to relate to established tests such as SeHCAT, as has recently been reported. ${ }^{29}$ These tests may eventually be found to have value, particularly in settings where SeHCAT is unavailable. SeHCAT, however, as a 7-day test, has the advantage that it can integrate multiple cycles of bile acid secretion and reabsorption and so be less affected by day-to-day variation and dietary effects. ${ }^{1}$ The limited support for a therapeutic trial, essentially only in Crohn's disease patients with ileal resection and negative inflammatory markers, indicates that, unlike previous suggestions, ${ }^{22}$ the value of a clear diagnosis before attempting treatment is recognised.

This survey shows that BASs, in particular colestyramine, are the main treatments used and that a good response rate is predicted in patients with severe disease, as shown by SeHCAT testing. This reflects the findings of our previous systematic review. ${ }^{10}$ Colesevelam, despite being more acceptable to many patients in tablet form, remains the second choice, but respondents recognised the need to try an alternative such as colesevelam or colestipol if treatment produced an incomplete response. Other aspects to highlight include starting with a low dose, preference for dosing last thing at night and considering a low fat diet to help reduce symptoms. ${ }^{30}$ Alternative drug therapies, including diets, are particularly relevant when the supply of medication is interrupted.

Our study is limited in that it reflects opinion only in the UK, but as we have discussed, there is greater use of SeHCAT and hence greater experience of BAD diagnosis and management than in most other countries. Other UK gastroenterologists who were not part of this review may have had different views. However, this review of expert opinion, particularly where there is consensus, should help inform future decisions and guidelines on BAD.

\section{Twitter Julian R F Walters@jrfw51}

Collaborators Members of UK Bile Acid Related Diarrhoea Network: Ayesha Akbar; Jervoise Andreyev; Richard Appleby; Ramesh Arasaradnam; Matthew Brookes; Anton Emmanuel; Adam Farmer; Alastair Forbes; Alex Ford; Subrata Ghosh; John Green; Ian Johnston; Matthew Kurien; John McLaughlin; Charlie Murray; Iain Murray; Jonathan Nolan; Sanjeev Pattni; David Sanders; Nidhi Sagar; and Julian Walters.

Contributors All authors designed the survey; JRFW: data collection and analysis; all authors: critical revision of the manuscript.

Funding The authors have not declared a specific grant for this research from any funding agency in the public, commercial or not-for-profit sectors.

Competing interests JRFW reports grants and personal fees from GE healthcare, grants from Novartis, personal fees from Pendopharm, personal fees from Prometheus, grants from Intercept, grants from ENYO, outside the submitted work. RA has received renumerations from GE healthcare for delivery of educational talks. HJNA has received remunerations as a member of advisory boards for GE Healthcare and Sanofi Aventis and has been provided by GE Healthcare with free SeHCAT capsules for completed and planned studies. 
Patient consent for publication Not required.

Provenance and peer review Not commissioned; externally peer reviewed.

Data availability statement Data are available on reasonable request.

Open access This is an open access article distributed in accordance with the Creative Commons Attribution Non Commercial (CC BY-NC 4.0) license, which permits others to distribute, remix, adapt, build upon this work noncommercially, and license their derivative works on different terms, provided the original work is properly cited, appropriate credit is given, any changes made indicated, and the use is noncommercial. See: http://creativecommons.org/licenses/by-nc/4. $0 /$.

\section{ORCID iDs}

Julian R F Walters http://orcid.org/0000-0001-9720-5835

Ramesh Arasaradnam http://orcid.org/0000-0002-2231-3062

\section{REFERENCES}

1 Walters JRF. Defining primary bile acid diarrhea: making the diagnosis and recognizing the disorder. Expert Rev Gastroenterol Hepatol 2010;4:561-7.

2 Mottacki N, Simrén M, Bajor A. Review article: bile acid diarrhoea - pathogenesis, diagnosis and management. Aliment Pharmacol Ther 2016;43:884-98.

3 Lacy BE, Mearin F, Chang L, et al. Bowel disorders. Gastroenterology 2016;150:1393-407.

4 Arasaradnam RP, Brown S, Forbes A, et al. Guidelines for the investigation of chronic diarrhoea in adults: British Society of gastroenterology, 3rd edition. Gut 2018;67:1380-99.

5 Hofmann AF. The syndrome of ileal disease and the broken enterohepatic circulation: cholerheic enteropathy. Gastroenterology 1967;52:752-7.

6 Hofmann AF, Poley JR. Cholestyramine treatment of diarrhea associated with ileal resection. N Engl J Med 1969;281:397402.

7 Fernández-Bañares F, Rosinach M, Piqueras M, et al. Randomised clinical trial: colestyramine vs. hydroxypropyl cellulose in patients with functional chronic watery diarrhoea. Aliment Pharmacol Ther 2015;41:1132-40.

8 Bajor A, Törnblom H, Rudling $\mathrm{M}$, et al. Increased colonic bile acid exposure: a relevant factor for symptoms and treatment in IBS. Gut 2015;64:84-92.

9 Beigel F, Teich N, Howaldt S, et al. Colesevelam for the treatment of bile acid malabsorption-associated diarrhea in patients with Crohn's disease: a randomized, double-blind, placebo-controlled study. J Crohns Colitis 2014;8:1471-9.

10 Wedlake L, A'Hern R, Russell D, et al. Systematic review: the prevalence of idiopathic bile acid malabsorption as diagnosed by SeHCAT scanning in patients with diarrhoeapredominant irritable bowel syndrome. Aliment Pharmacol Ther 2009;30:707-17.

11 Slattery SA, Niaz O, Aziz Q, et al. Systematic review with meta-analysis: the prevalence of bile acid malabsorption in the irritable bowel syndrome with diarrhoea. Aliment Pharmacol Ther 2015;42:3-11.

12 Walters JRF, Tasleem AM, Omer OS, et al. A new mechanism for bile acid diarrhea: defective feedback inhibition of bile acid biosynthesis. Clin Gastroenterol Hepatol 2009;7:1189-94.
13 Bannaga A, Kelman L, O'Connor M, et al. How bad is bile acid diarrhoea: an online survey of patient-reported symptoms and outcomes. BMJ Open Gastroenterol 2017;4:e000116.

14 Pattni SS, Brydon WG, Dew T, et al. Fibroblast growth factor 19 in patients with bile acid diarrhoea: a prospective comparison of FGF19 serum assay and SeHCAT retention. Aliment Pharmacol Ther 2013;38:967-76.

15 Valentin N, Camilleri M, Altayar O, et al. Biomarkers for bile acid diarrhoea in functional bowel disorder with diarrhoea: a systematic review and meta-analysis. Gut 2016;65:1951-9.

16 Vijayvargiya P, Camilleri M. Current practice in the diagnosis of bile acid diarrhea. Gastroenterology 2019;156:1233-8.

17 Boyd GS, Merrick MV, Monks R, et al. Se-75-labeled bile acid analogs, new radiopharmaceuticals for investigating the enterohepatic circulation. J Nucl Med 1981;22:720-5.

18 Sciarretta G, Fagioli G, Furno A, et al. 75Se HCAT test in the detection of bile acid malabsorption in functional diarrhoea and its correlation with small bowel transit. Gut 1987;28:9705 .

19 Orekoya O, McLaughlin J, Leitao E, et al. Quantifying bile acid malabsorption helps predict response and tailor sequestrant therapy. Clin Med 2015;15:252-7.

20 Gupta A, Muls AC, Lalji A, et al. Outcomes from treating bile acid malabsorption using a multidisciplinary approach. Support Care Cancer 2015;23:2881-90.

21 Walters JRF, Johnston IM, Nolan JD, et al. The response of patients with bile acid diarrhoea to the farnesoid X receptor agonist obeticholic acid. Aliment Pharmacol Ther 2015;41:5464.

22 Riemsma R, Al M, Corro Ramos I, et al. SeHCAT [tauroselcholic (selenium-75) acid] for the investigation of bile acid malabsorption and measurement of bile acid pool loss: a systematic review and cost-effectiveness analysis. Health Technol Assess 2013;17:1-236.

23 Summers JA, Peacock J, Coker B, et al. Multicentre prospective survey of SeHCAT provision and practice in the UK. BMJ Open Gastroenterol 2016;3:e00091.

24 Fromm H, Malavolti M. Bile acid-induced diarrhoea. Clin Gastroenterol 1986;15:567-82.

25 Smith MJ, Cherian P, Raju GS, et al. Bile acid malabsorption in persistent diarrhoea. J R Coll Physicians Lond 2000;34:44851.

26 Nyhlin H, Merrick MV, Eastwood MA. Bile acid malabsorption in Crohn's disease and indications for its assessment using SeHCAT. Gut 1994;35:90-3.

27 Turner JM, Pattni SS, Appleby RN, et al. A positive SeHCAT test results in fewer subsequent investigations in patients with chronic diarrhoea. Frontline Gastroenterol 2017;8:279-83.

28 Fernandes DCR, Poon D, White LL, et al. What is the cost of delayed diagnosis of bile acid malabsorption and bile acid diarrhoea? Frontline Gastroenterol 2019;10:72-6.

29 Walters JR, Sagar N, Duboc H, et al. Mo1542 - primary bile acids in a single fecal sample for the diagnosis of bile acid diarrhea: relationship to Sehcat testing. Gastroenterology 2019;156:S-774-774.

30 Watson L, Lalji A, Bodla S, et al. Management of bile acid malabsorption using low-fat dietary interventions: a useful strategy applicable to some patients with diarrhoeapredominant irritable bowel syndrome? Clin Med $2015 ; 15: 536-40$. 